\title{
Supportive Cancer Chemotherapy
}

National Cancer Institute

\section{Source}

National Cancer Institute. Supportive Cancer Chemotherapy. NCI Thesaurus. Code C15981.

Treatment designed to improve the health of a chemotherapy patient but that does not directly affect the tumor. 